\title{
Lift-out and in-situ STM-TEM Studies of Individual GaN Nanowires
}

\author{
Mitra L. Taheri*, Blake Simpkins**, Pehr Pehrsson** and Rhonda M. Stroud*
}

*Materials Science and Technology Division, Naval Research Laboratory, Washington, D.C. 20375

**Chemistry Division, Naval Research Laboratory, Washington, D.C. 20375

GaN nanowires are a crucial component in various nanoscale optoelectronic devices, such as blue light-emitting diodes and short-wavelength ultraviolet nanolasers [1-3]. Grain boundaries, extended defects and impurity incorporation from heterogeneous catalysts used to initiate wire growth can all negatively impact a wire's electronic properties. To understand the specific effects of microstructure and composition on electrical properties, these must be correlated for individual nanowires. Recent measurements [4,5] demonstrate the relationship between electrical resistance and wire microstructure for B-doped SiN nanowires. Quantitative results, however, were limited due to inefficient acquisition of single nanowires for scanning tunneling microscopy (STM) measurements [4]. Here we present a novel and convenient method of collecting single nanowires, and subsequent correlation of electrical and microstructural properties.

We synthesized Ni-catalyzed and $\mathrm{Ga}\left(\mathrm{NO}_{3}\right)_{3}$-catalyzed $\mathrm{GaN}$ nanowires on $\mathrm{Si}$ substrates by atmospheric chemical vapor deposition (CVD) [6]. To extract individual $\mathrm{GaN}$ nanowires from the dense batch of wires on the $\mathrm{Si}$ substrate, we used an Ascend Extreme Access ${ }^{\mathrm{TM}}$ in situ lift-out system mounted in a FEI Nova $600^{\mathrm{TM}}$ Dual Beam focused ion beam (FIB) workstation. A Nanofactory ${ }^{\mathrm{TM}}$ STM-TEM holder [7] allowed us to characterize, in-situ, the structures and electrical properties of the extracted wires with a JEOL ${ }^{\mathrm{TM}} 2200 \mathrm{FS}$ transmission electron microscope (TEM) (Fig. 1a). This holder can accommodate samples mounted on $\mathrm{Au}$ wires or conventional TEM grids. We extracted nanowires using both $\mathrm{Cu}$ microtweezer grids, called End-Effectors ${ }^{\mathrm{TM}}$ (Ascend Instruments, LLC) and Au wires (Fig. 1). The use of the End-Effectors provided greater flexibility in site-specific microstructural analysis because of their compatibility with conventional TEM holders.

Electron diffraction studies showed that both Ni-catalyzed and $\mathrm{Ga}\left(\mathrm{NO}_{3}\right)_{3}$-catalyzed wires exhibited a polycrystalline wurtzite structure. The Ni-catalyzed wires possessed strong [011] growth texture, in contrast to the [111] and [001] preferred orientations in the $\mathrm{Ga}\left(\mathrm{NO}_{3}\right)_{3}$-catalyzed wires [7]. Point-contact I-V curves (Fig. 3) were obtained using the STM system to quantitatively assess wire resistance. TEM imaging and STEM-based energy dispersive x-ray spectroscopy will be used to determine trends in structure and composition at specific points on a wire. Trends in crystal size, growth orientation and composition along the wire will be compared to IV curves of the same wires. In some cases, the lift-out preserved the catalyst-wire interface, permitting determination of relationships between the catalyst morphology and wire microstructure [8].

\section{References}

[1] Xia et al., Adv. Mater. 15, No.5, (2003) 353.

[2] E.A. Stach et al., Nano Lett 3, No. 6, (2003) 867.

[3] P. Yang et al., Nature Materials 3 (2004) 524.

[4] D.D.D. Ma et al., Science 299 (2003) 1874.

[5] D.D.D. Ma et al., App. Phys. Lett., 79, No. 15 (2001) 2468.

[6] B. Simpkins et al., Journal of Crystal Growth, in press.

[7] K. Svensson et al., Review of Scientific Instruments, 74, 11, (2003), 4945. 
[8] This work was funded by The Naval Research Lab and The Office of Naval Research. Authors M. Taheri and B. Simpkins thank the NRC Postdoctoral Fellowship Program for financial support. Oleg Lourie of Gatan and Ludvig de Knoop of Nanofactory are gratefully acknowledged for their assistance with our instrumentation.
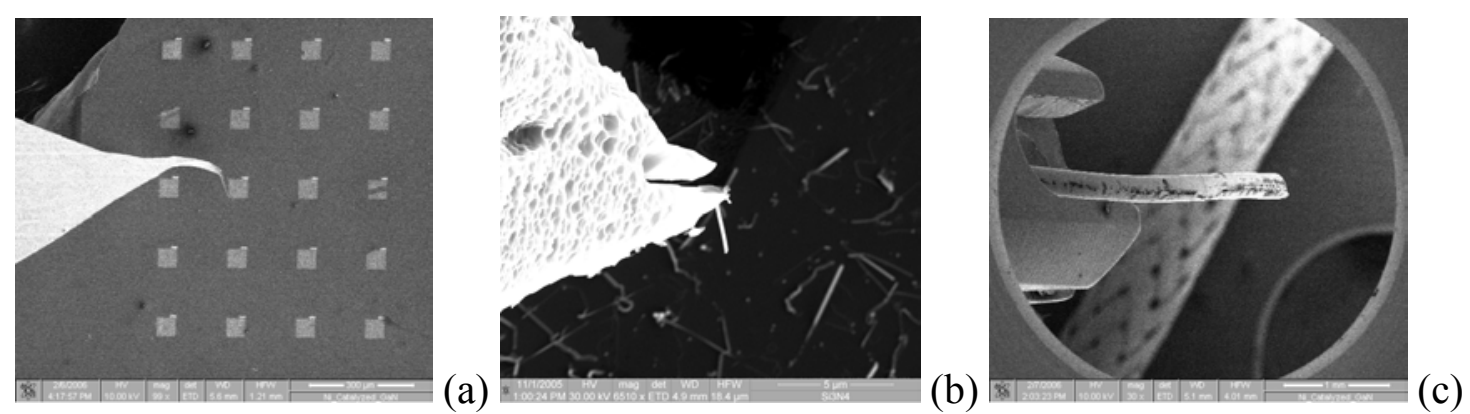

FIG.1. Secondary electron (SE) images of (a) a $\mathrm{Cu}$ end-effector approaching a sample area of interest; (b) a $\mathrm{GaN}$ nanowire held by electrostatic force on a 'microtweezer'; (c) an Au wire in an Ascend carrier.
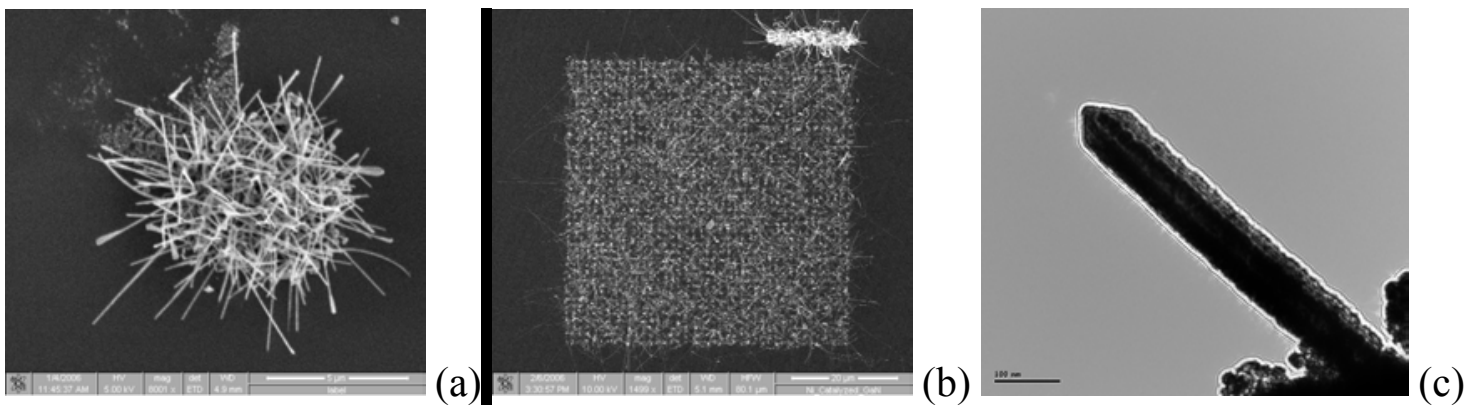

FIG.2. SE images of (a) $\mathrm{Ga}\left(\mathrm{NO}_{3}\right)_{3}$-catalyzed $\mathrm{GaN}$ wires at a nucleation site, and (b) Ni-catalyzed $\mathrm{GaN}$ wires at a nucleation grid site; (c) Bright-field TEM image of an extracted GaN nanowire.

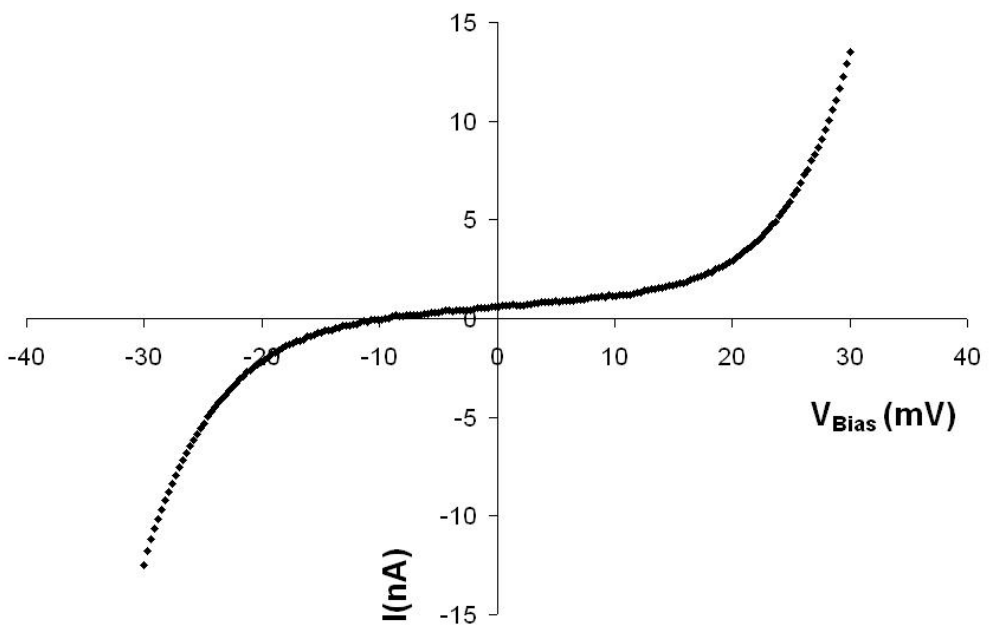

FIG.3. I-V curve of a GaN nanowire using the Nanofactory ${ }^{\mathrm{TM}}$ STM/TEM apparatus. 